\title{
Nematic braids: topological invariants and rewiring of disclinations
}

\author{
Simon Čopar ${ }^{1}$ and Slobodan Žumer ${ }^{1,2}$ \\ ${ }^{1}$ Faculty of Mathematics and Physics, University of Ljubljana, Jadranska 19, 1000 Ljubljana, Slovenia \\ ${ }^{2}$ Jožef Stefan Institute, Jamova 39, 1000 Ljubljana, Slovenia
}

(Dated: October 23, 2018)

\begin{abstract}
The conventional topological description given by the fundamental group of nematic order parameter does not adequately explain the entangled defect line structures that have been observed in nematic colloids. We introduce a new topological invariant, the self-linking number, that enables a complete classification of entangled defect line structures in general nematics, even without particles, and demonstrate our formalism using colloidal dimers, for which entangled structures have been previously observed. We also unveil a simple rewiring scheme for the orthogonal crossing of two $-1 / 2$ disclinations, based on a tetrahedral rotation of two relevant disclination segments, that allows us to predict possible nematic braids and calculate their self-linking numbers.
\end{abstract}

PACS numbers: 61.30.Dk,61.30.Jf,82.70.Dd

Depending on the temperature and their molecular properties, nematogenic media can form isotropic, nematic, chiral nematic or even blue phases, each characterized by a specific orientational ordering of the constituent molecules. Their anisotropic nature allows the formation of disclinations that can be stabilized by geometric or intrinsic constraints. Recently, a lot of progress has been made on the stabilization and manipulation of disclinations by using dispersions of colloidal particles [14 or confinement to porous networks [5]. In nematic dispersions, the anisotropic inter-particle interactions mediated by elastic deformations and defects lead to diverse colloidal structures that promote self-assembly and offer great potential for photonics and plasmonics [6]. Nematic braids are disclination networks where defect loops are not localized around one particle, but instead entangle clusters of particles [7, 8]. The existence of entangled structures was first proposed based on the results of numerical simulations [9, 10]. They have since been observed experimentally and their stability has been extensively analyzed [7, 11. These structures are not sufficiently well described by the theory developed for simple nematic defects 12 and a complete theoretical understanding is still lacking. Nematic braids may also include knots and links [13, otherwise seen in the physics of polymers [14, DNA [15 17] and knotted light [18. Easy experimental observation of nematic disclination networks, and their rewiring, knotting and linking by laser tweezers [7, 13, places nematic braids as a primary template for the study of nontrivial topology in physical systems.

Nematic braids stabilized by homeotropic particles consist of closed $-1 / 2$ disclination loops. To fully describe a single disclination loop, we generalize the mathematical notion of a loop by introducing the self-linking number, which counts how many times the cross section of the disclination turns during a complete loop. This invariant applies to elastic loops, DNA loops 17 and other fields, but in the case of a $-1 / 2$ nematic disclination, due to its intrinsic three-fold symmetry, it assumes specific fractional values, similar to flux discretization in the fractional quantum Hall effect [19].

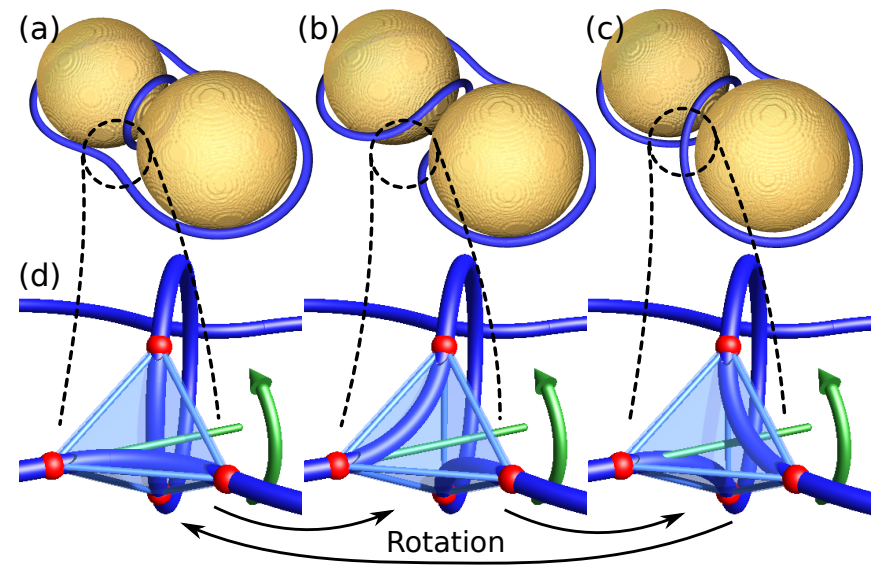

FIG. 1. Rewiring sites of different dimer structures. (a-c) Theta structure and two chiral omega structures (simulations by M. Ravnik [7]). Rewiring sites are marked and paired with corresponding idealized structures. (d) The three conformations of a disclination crossing with tetrahedral symmetry. Rewiring is performed by rotating around a $C_{3}$ symmetry axis of the tetrahedron.

For the investigation of the self-linking number, we chose a colloidal dimer consisting of two spherical particles with strong homeotropic anchoring, confined to a homogeneous planar nematic cell [7. Depending on the particle size, confinement and initial conditions, the particles can interact by arranging themselves into dipolar or quadrupolar structures [2], or they can be bound by $-1 / 2$ disclination loops shared between both particles [11. The homogeneous director field environment energetically disfavors linking and knotting, which reveals the more basic rewiring properties of entangled states.

We demonstrate that differences between dimer structures are localized to tetrahedral regions around crossings of disclinations (Fig. 1), from which we derive rules for calculating the self-linking number and classifing all dimer structures. The rewiring rules apply to structures involving $-1 / 2$ disclinations in any confinement and can be used to predict and design nematic braids consisting 
of complex linked and knotted loops.

We start by examining the similarities of two dimer disclination configurations [11]. The "entangled hyperbolic defect structure" (referred to as the theta structure from here on) is the only dimer structure with space inversion symmetry and consists of two perpendicular loops, one encircling both particles and the other placed symmetrically between them (Fig. 1a). The "omega structure", on the other hand, consists of a single loop wrapped around both particles and has two chiral isomers (Fig. 1p,c). All three structures have similar director field and line geometry at the far ends of the colloidal particles and along the vertical axis (Fig. 5 in [11). They only differ in the way the left arc, right arc and central loop meet between the particles (see the encircled areas on Fig. 1 1 -c). The conversion of one structure into another is achieved by rewiring the crossing, while leaving the remote field intact, which requires a cutting of disclinations. The resulting four endpoints define a tetrahedron that encloses the rewiring site. Inside the tetrahedron, two perpendicular disclination segments connect pairs of the four vertices (Fig. 11). Experimentally, rewiring is achieved by local laser melting of a nematic [13].

The three-fold symmetry of $-1 / 2$ disclinations (Fig. 2) entering the tetrahedron through the vertices coincides with the $C_{3}$ tetrahedral symmetry axes. The director field inside the tetrahedron has intrinsic dihedral symmetry $\left(D_{2 d}\right)$, ensured by the relative positioning of the disclinations. Due to this symmetry and the profile of the disclinations, the director field stands perpendicularly to all the faces of the tetrahedron and makes hyperbolic turns at all the edges, thus completing the full tetrahedral symmetry of the director field on the surface of the tetrahedron. Consequently, rotations from the tetrahedral symmetry group preserve the continuity of the disclination lines and the surrounding director field and therefore always generate physically possible structures. As the disclination segments inside the tetrahedron have lower symmetry $\left(D_{2 d}\right)$ than the field on its surface, rotations around a chosen $C_{3}$ symmetry axis generate 3 distinct configurations of disclinations, depicted in Fig. 1 $1 \mathrm{~d}$.

The real director field deviates from perfect tetrahedral symmetry in order to accommodate the proximity of the particles and to minimize the free energy. However, it only differs from the idealization by a continuous transformation, so the topological invariants are not affected. In further derivations, we assume this symmetry to be exact.

The director field surrounding a disclination may rotate around the disclination line tangent. In a closed loop, rotations are restricted by the fact that the director field must be continuous. The loop, together with the orientation of its cross-section, can be described mathematically by a ribbon (Fig. 22). A ribbon can be assigned a self-linking number, $S l$, a topological invariant that labels how many times it turns around its tangent in the course of one loop. Because of the three-fold symmetry of disclinations, the self-linking number is not restricted to

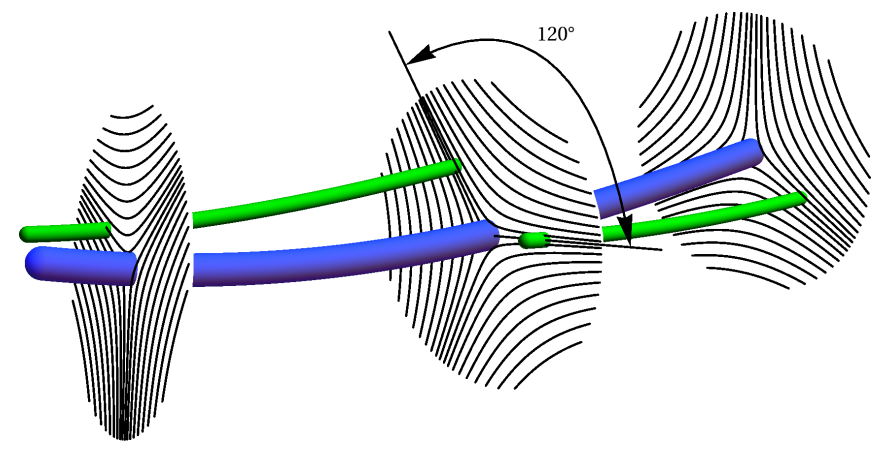

FIG. 2. To describe a disclination line, we introduce a ribbon, defined by an axis curve and a secondary curve that follows the orientation of the field cross section of the disclination. The ribbon may reconnect with itself with an offset angle of $\pm 120^{\circ}$ while keeping the director field continuous, due to the symmetry of its cross section.

integers, but can assume any third-integer value (Fig. 2). Using of Călugăreanu theorem, we can decompose the self-linking number into writhe and twist [20, 21,

$$
S l=W r+T w .
$$

Writhe depends on how the loop changes direction in space, while twist contains information about the local torsion of the ribbon around its axis. Consider the theta structure (Fig. 19). Up to an arbitrary homotopic transformation, the structure is completely symmetric and both loops are planar. Both twist and writhe therefore equal zero, which can be verified using the corresponding Gauss integral definitions [20]. Tetrahedral rotation of a portion of the ribbon does not change the twist, as it is defined as an integral of local twist density, which is preserved by rigid transformations. As we have shown that the theta structure has zero twist, the same holds for all entangled dimer structures. It follows from Eq. (1) that in this idealization the self-linking number equals the writhe. The physics of nematic liquid crystals is hidden in the transformation rules for rewiring and is not involved in the computation of writhe, which only depends on the disclination loop geometry. In practice, disclination loops do not necessarily have zero twist, but this can always be changed by continuous transformations that preserve the topology of the structure. The twist and writhe convert into each other under such transformations, but their sum remains equal to the self-linking number, calculated in our idealized case.

Since the writhe only depends on the axis curve of a ribbon, ordinary loops can be used instead of ribbons. We use the tantrix representation: the loop is mapped to the unit sphere of tangents. Fuller's formula [16] expresses writhe in terms of the spherical area $A$ enclosed by the tangent indicatrix (tantrix) loop. The writhe given by this formula has modulo 2 ambiguity because full $4 \pi$ wraps do not change the tantrix loop,

$$
W r=\frac{A}{2 \pi}-1 \bmod 2
$$


(a)

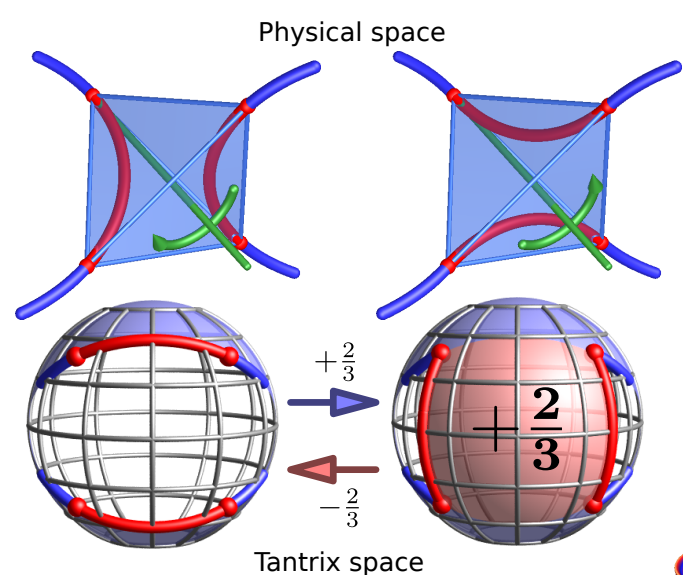

(b)



FIG. 3. (a) Tetrahedral rotation changes the positions of two disclination line segments, which also changes the curve, traced by the tangent on the unit sphere. The spherical area traced by this curve changes by $\pm 4 \pi / 6$ (shown in red), which is directly related to the $\pm 2 / 3$ change in writhe and consequently, the self-linking number. (b) Schematic depiction of dimer structures and transformations between them. A tetrahedral rotation changes the writhe by $\pm 2 / 3$ if parametrizations of initial and final structure are the same. By varying the parametrization, we can calculate writhes of all different dimer structures. Depicted here are the theta, chiral omega and figure-eight structures and the pair of Saturn rings, with their respective self-linking numbers. Structures that consist of two loops are shown with both possible choices of parametrization. Orientations of loop parametrizations are indicated by arrows.

The tetrahedral rotations change which pairs of the vertices are connected by segments of the disclination loop. Each loop segment is planar and maps to a great circle arc on the tantrix sphere (Fig. 3a). The endpoints of these arcs are the tangents through the vertices of the tetrahedron, which form a square on the tantrix sphere. Rewiring induced by the tetrahedral rotation changes the enclosed area by $\mp 4 \pi / 6$, which by Fuller's formula (2) corresponds to a $\pm 2 / 3$ change in writhe. This is consistent with the restriction of the self-linking number to thirds, imposed by the symmetry of $-1 / 2$ disclinations (Fig. 2). It can be shown that the modulo 2 ambiguity in Eq. (2) can be dropped in our case (see appendix). As the tangents flip sign if the parametrization of a curve is reversed, the $\pm 2 / 3$ change in writhe is correct only for tetrahedral rotations that preserve continuous parametrization of the loops. If this is not the case, the change in writhe can be calculated by finding a succession of multiple rewirings that result in the same structure (Fig. 3b).

We can generalize the notion of writhe for a union of two or more loops $A_{i}$ that may or may not be linked. The Gauss integral that defines writhe decomposes into writhes of individual loops, which equal self-linking numbers, $S l\left(A_{i}\right)$, and linking numbers $L k\left(A_{i}, A_{j}\right)$ between pairs of loops (see appendix)

$$
W r\left(A_{0} \cup \cdots \cup A_{n}\right)=\sum_{i} S l\left(A_{i}\right)+2 \sum_{i>j} L k\left(A_{i}, A_{j}\right) .
$$

Combining this with the fact that every parametrization- preserving rewiring changes the total writhe by $\pm 2 / 3$ and changes the number of loops, $n$, by one 22, we can write a conservation law

$$
\frac{3}{2}\left(\sum_{i}^{n} S l\left(A_{i}\right)+2 \sum_{i>j}^{n} L k\left(A_{i}, A_{j}\right)\right)+n=q \bmod 2 .
$$

Linking numbers are integers with ambiguously defined sign and the number of loops may either increase or decrease by one, hence the modulo 2 . This relation is a generalized conservation of topological charge $q[12$ and reflects the fact that, due to the presence of line defects, only the even/odd parity of $q$ is conserved [12, 23. The interpretation of $q$ as the topological charge can be justified with an example. Consider $q$ homeotropic spherical particles in a planar nematic cell, each with its own Saturn ring loop 22. Such a system satisfies the above equation as it contains $n=q$ unlinked loops with $S l=0$. Entangled structures can be reached by applying successive tetrahedral rotations, which preserve both the left side of Eq. (4) and the topological charge.

The derived formalism can be demonstrated using our dimer structures. There are two rewiring sites situated symmetrically between the colloidal particles. The 3 . $3=9$ possible structures consist of one theta structure, two equivalent structures with disjoint Saturn rings, two equivalent pairs of chiral omega structures and a pair of chiral figure-eight structures. The theta structure has zero self-linking number and the others are reached by successive tetrahedral rotations. The structures with one loop have self-linking number $\pm 2 / 3$ while the structures 
with two loops have both self-linking numbers equal to zero, which agrees with the conservation law (4). The results are shown in Fig. 3 p.

The derived conservation law (4) holds for a set of multiple linked loops as a whole. Individual constituent loops have a self-linking number of the form $p / 3$, where $p$ is even if an even number of disclinations pass through the loop and odd in the converse case. This can be shown by choosing an idealized model of a director field representing such loop and determining mathematically which element of the fundamental group the given director field represents [12, 23]. The calculation is carried out in appendix.

We have shown that any rewiring of two orthogonally crossing $-1 / 2$ disclinations is possible, as the topological requirements are satisfied entirely by the changes in the self-linking and linking numbers caused by the application of the tetrahedral rotations. In contrast, $+1 / 2$ disclinations cannot form rich entangled structures, as they only allow integer self-linking numbers. Under the restriction that only $-1 / 2$ disclinations are present, the self-linking numbers of the loops are topological invariants, coupled with surrounding topological charges by a conservation law. In confined, chiral and field-affected environments, however, the type of the disclination profile may vary between $\pm 1 / 2$ and twist disclinations [24]. Our findings do not apply directly to such cases, as the self-linking number is ill-defined if the disclination crosssection does not have constant symmetry.

Our work introduces two important advances in the theoretical understanding of nematic braids. By combining the formalism of differential geometry with the characteristics of nematic defects, we are able to show that the self-linking number is a topological invariant of $-1 / 2$ disclination loops that successfully differentiates between the loops and ensures the conservation of topological charge. On the other hand, our explanation of local rewiring by tetrahedral rotations gives a qualitative three-dimensional image of disclinations and resolves the behavior of director in complex disclination loop networks seen in experiments and simulations [8, 10, 13. The richness of entangled structures increases with the number of available rewiring sites, which are more abundant in chiral systems [4, 13. The rewiring rules classify the set of possible braids and allow a transparent design of new structures and guidance of their experimental realization. Provided symmetry-driven rewiring rules similar to our tetrahedral rotations exist, our formalism can be extended to systems with different line defects, crossing geometries [25], or any system with a well-defined selflinking number (e.g. loop DNA [17, 26]).

We thank T. Lubensky and R. Kamien for helpful discussions regarding the topological interpretation of the results. We acknowledge support from the Slovenian Research Agency (research program P1-0099 and project J1-2335) and the NAMASTE Centre of Excellence.

\section{APPENDIX}

In the following sections, we supplement the main article with a review of the basic knowledge of differential geometry of loops and ribbons needed to describe general structures consisting of nematic disclinations. All curves are assumed to be regularly parametrized, so that their tangents are uniquely defined.

\section{Appendix A: Gauss map}

Consider two nonintersecting curve segments $A$ and $B$ with parameters $s$ and $s^{\prime}$. Each pair of points from the two segments define a chord. For a given parametrization, we can define a Gauss map that assigns a direction of the chord to each pair of points (Fig. 4 a). This maps from the set of chords (chord manifold) to the unit sphere 20, 27.

$$
\left(s, s^{\prime}\right) \mapsto \frac{\mathbf{r}_{A}(s)-\mathbf{r}_{B}\left(s^{\prime}\right)}{\left|\mathbf{r}_{A}(s)-\mathbf{r}_{B}\left(s^{\prime}\right)\right|} \in S^{2}
$$

A rectangle in parameter space is mapped to a patch on the unit sphere (Fig. 4). The area of the patch is obtained by integrating the Jacobian of the Gauss map, which yields a Gauss integral.

$\mathcal{G}(A, B)=\frac{1}{4 \pi} \iint \mathbf{t}_{A}(s) \times \mathbf{t}_{B}\left(s^{\prime}\right) \cdot \frac{\mathbf{r}_{A}(s)-\mathbf{r}_{B}\left(s^{\prime}\right)}{\left|\mathbf{r}_{A}(s)-\mathbf{r}_{B}\left(s^{\prime}\right)\right|^{3}} \mathrm{~d} s \mathrm{~d} s^{\prime}$

From above definition, it is evident that the Gauss integral is commutative and bilinear under union of curve segments (Fig. 4 F):

$$
\mathcal{G}(A, B \cup C)=\mathcal{G}(A, B)+\mathcal{G}(A, C) \quad .
$$

If the curves $A$ and $B$ are closed (we will refer to closed curves as loops), the parameters are cyclic and therefore the chord manifold has no boundary (it has topology of a torus). The patch on the unit sphere also has no boundary, so its area can only be an integer multiple of $4 \pi$, depending on how many times the map wraps the sphere. The Gauss integral of two loops defines the linking number $L k(A, B)=\mathcal{G}(A, B)$, which is the number of times the loops pass through each other [20, 21, 28].

Instead of a simple loop, consider a ribbon, an infinitesimally narrow two-dimensional strip, seamlessly closed into a loop. A ribbon can be specified by its two boundary loops: an axis loop and a secondary loop that differs from the axis loop by a infinitesimal displacement [27. A ribbon can twist around its tangent, which is suitable for description of $-1 / 2$ nematic disclination lines. The linking number of the axis loop and the secondary loop labels the ribbons according to the number of twists incorporated in the loop and is invariant under continuous transformations. To differentiate the linking number of a ribbon from the linking number of two arbitrary curves, we will label it with symbol $S l$ and call it a self-linking 




(c)

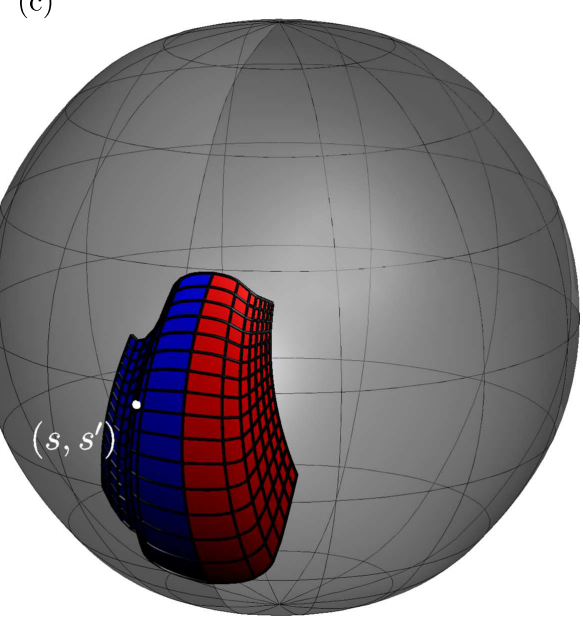

unit sphere

FIG. 4. (a) Two curve segments and a few chords spanning between them. Their directions are marked with arrows. (b) For two curves in space, a chord between two points is defined for every pair of parameters $\left(s, s^{\prime}\right)$. The chord directions continuously vary with the parameters. (c) Directions of chords lie on a patch on the unit sphere. Gauss integral calculates the area of this patch. One curve is represented as a union of two segments (red and blue), which manifests as segmentation of the patch on the unit sphere. Gauss integral is bilinear because areas of these patches are additive.

number (this term is used in the field of theoretical geometry in a narrower context [29]). Even though both axis loop and secondary loop of the ribbon have to be continous for the self-linking number to be defined, we can assign fractional self-linking numbers to a disclination loop, if the ribbon representing the disclination line runs around the disclination loop multiple times. In the case of nematics, the three-fold symmetry of $-1 / 2$ disclinations restricts the linking number to third-integer values.

The Călugăreanu theorem (1) decomposes the Selflinking number into twist that can be seen as contribution of locally distributed torsion and writhe that measures nonplanarity of the curve. The writhe is a Gauss integral of the ribbon's axis loop with itself, $W r(A)=\mathcal{G}(A, A)$. The chord manifold in this case is not a torus but an annulus, which has two boundaries, so unlike the linking number, the writhe can assume any value [27. The twist, on the other hand, is defined as a single integral along the loop and the integrand can be interpreted as a local twist density [20].

$$
T w=\frac{1}{2 \pi} \oint \mathbf{t}(s) \cdot\left(\mathbf{u}(s) \times \partial_{s} \mathbf{u}(s)\right) \mathrm{d} s
$$

$\mathbf{u}$ is a normalized vector, perpendicular to the axis loop, that defines the orientation of the ribbon's cross section [20]. Writhe and twist are not topological invariants, but the self-linking number is, which means that homotopic transformations of the ribbon only convert between writhe and twist, preserving their sum.

\section{Appendix B: Writhe and the tetrahedral rotations}

We have shown that the tetrahedral rotation changes the writhe by $\pm 2 / 3$. We used Fuller's formula (2), that does not take into account the entire Gauss integral, but only maps the boundaries of the chord manifold. This technique misses full $4 \pi$ wraps of the sphere, so the writhe is undetermined up to an integer multiple of 2 . With a reasoning described below, we show that the change of writhe by the tetrahedral rotations is exactly $\pm 2 / 3$, without an undefined additive offset.

We can represent the loop as a union of two loop segments inside the tetrahedron $A$ and everything else $X$ (Fig. 5p). Using bilinearity (Eq. A3) we can expand the result to Gauss integrals between combinations of segments.

$$
W r(A \cup X)=\mathcal{G}(A, A)+\mathcal{G}(X, X)+2 \mathcal{G}(A, X)
$$

The tetrahedral rotation rigidly rotates the segments $A$ and preserves $X$, so the only affected term is the last one. This term is a mapping from a set of chords spanning between $A$ and $X$ to the space of their directions. In our case, the tetrahedron also contains nematic director field, which means no other disclinations may be present inside it. If the rotation is performed continuously, no disclination lines are crossed by the rotating segments in the process. We can therefore make a homotopic transformation that stretches every part of the loop outside the tetrahedron towards the infinity (Fig $5 \mathrm{~b}$ ). The chords extending from the loop segments $A$ to the points at infinity $X$ do not change direction under finite movements of the loop segments $A$. The only chords that change are those 


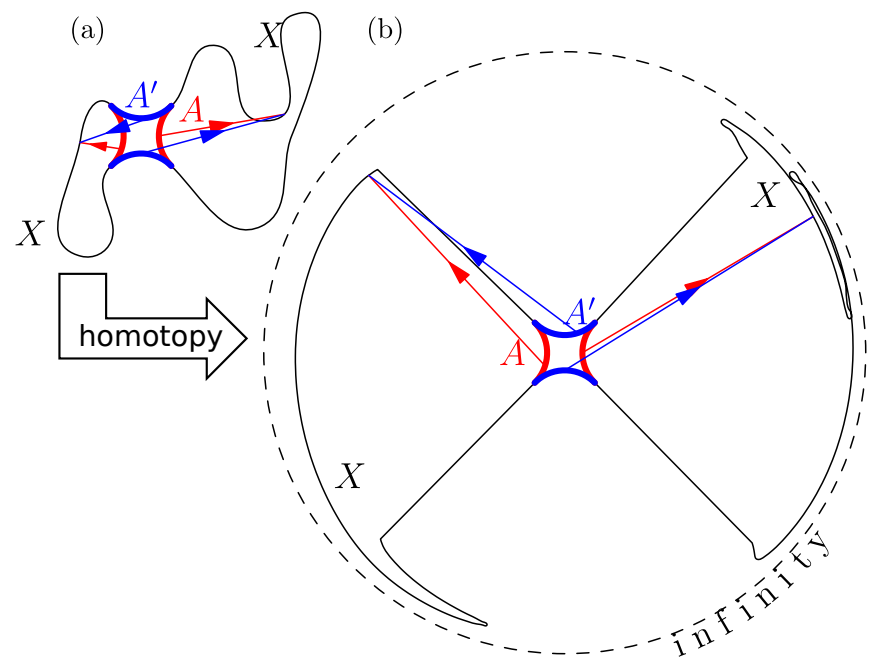

FIG. 5. (a) A tetrahedral rotation transforms segments $A$ into $A^{\prime}$, which only influences the chords between these segments and the rest of the loop $X$. (b) We can perform a homotopic transformation that pushes everything but the rewiring site to the infinity. In this limit, rewiring for all the curves looks exactly the same and the chords connected to the infinity do not change direction with rewiring. Note that on this sketch, the chords differ because the curve is still at a finite distance from the rewiring site.

extending from the loop segments to the straight paths connecting the tetrahedral vertices to the infinity. These paths are independent from the rest of the loop $(X)$, so the change in writhe for tetrahedral rotation is universal. The largest angle for which the chords can change their direction is $\pi / 3$, which happens for the limiting case at the endpoints of the disclination segments. Together with the fact that most chords do not change at all, this is not enough to change the area of the patch on the unit sphere for $4 \pi$. Our result that rewiring changes the writhe for $\pm 2 / 3$ is therefore correct, without the modulo 2 ambiguity suggested by Fuller's formula.

\section{Appendix C: Homotopy of disclination loops with nonzero self-linking number}

To investigate the topological properties of loops with nonzero self-linking number, we have the liberty to choose any configuration with desired self-linking number, as homotopic transformations do not influence the result. We choose a planar ring-shaped disclination loop with radius 1 and parametrize the surrounding space in toroidal coordinates with the main angle $\psi$ and inner angle $\theta$. The basis vectors of a crossection perpendicular to the disclination line are $\hat{e}_{\psi}=\cos \psi \hat{e}_{x}+\sin \psi \hat{e}_{y}$ and $\hat{e}_{z}$.

We are interested in disclination lines with winding number $-1 / 2$, which is the number of times the director rotates when we encircle the loop. We start by choosing director field for the cross section at $\psi=0$.

$$
\mathbf{n}(\theta, 0)=\cos \left(-\frac{\theta}{2}\right) \hat{e}_{\psi=0}+\sin \left(-\frac{\theta}{2}\right) \hat{e}_{z}
$$

To describe a disclination loop with nonzero self-linking number $S l$, this cross section must rotate rigidly $S l$-times when $\psi$ increases to $2 \pi$. By evaluating these rotations, we obtain full specification of the director field around the disclination loop.

$$
\mathbf{n}(\theta, \psi)=\cos \left(\frac{1}{2}(\theta-3 S l \psi)\right) \hat{e}_{\psi}-\sin \left(\frac{1}{2}(\theta-3 S l \psi)\right) \hat{e}_{z}
$$

To describe the topology of the director field on a torus, we need two winding numbers 23 . The winding number of the small toroidal circle we already know: it equals $-1 / 2$. The winding number on the great toroidal circle is determined by the mapping $\mathbf{n}(0, \psi)$, where we fixed the value of $\theta=0$, and measures the homotopy class of the director field encircled by our disclination loop.

$$
\mathbf{n}(0, \psi)=\left(\cos \left(\frac{3}{2} S l \psi\right) \cos \psi, \cos \left(\frac{3}{2} S l \psi\right) \sin \psi, \sin \left(\frac{3}{2} S l \psi\right)\right)
$$

Fundamental group of nematic order parameter only has two elements: integer winding numbers correspond to a director field, homotopic to defect-free field and halfinteger, which corresponds to defect lines. We know that in the first case, the vector representation of the director field is continuous and in the latter case, it has a sign discontinuity between $\mathbf{n}(0,0)$ and $\mathbf{n}(0,2 \pi)$. Assuming $S l=p / 3$, we get the condition $\cos (p \pi)= \pm 1$. Self-linking numbers $S l=\left\{\ldots,-\frac{2}{3}, 0, \frac{2}{3}, \frac{4}{3}, \ldots\right\}$ therefore correspond to director field, homotopic to defectfree field, which is achieved if even number of disclination lines pass through our loop. Self-linking numbers $S l=\left\{\ldots,-1,-\frac{1}{3}, \frac{1}{3}, 1, \ldots\right\}$ arise in case odd number of disclination lines pass through our loop. For two loops, their linking number measures how many times they pass through each other and therefore chooses between two distinct sets of possible self-linking numbers described above.
[1] P. Poulin, H. Stark, T. C. Lubensky, and D. A. Weitz, Science 275, 1770 (1997).

[2] I. Muševič, M. Škarabot, U. Tkalec, M. Ravnik, and S. Žumer, Science 18, 954 (2006).

[3] C. P. Lapointe, T. G. Mason, and I. I. Smalyukh, Science 326, 20 (2009).
[4] N. Hijnen, T. A. Wood, D. Wilson, and P. Clegg, Langmuir 26, 13502 (2010).

[5] T. Araki, M. Buscaglia, T. Bellini, and H. Tanaka, Nat. Mat. 10, 303 (2011).

[6] J. D. Joannopoulos, S. G. Johnson, J. N. Winn, and R. D. Meade, Photonic Crystals: Molding the Flow of 
Light, 2nd ed. (Princeton University Press, 2008).

[7] M. Ravnik, M. Škarabot, S. Žumer, U. Tkalec, I. Poberaj, D. Babič, N. Osterman, and I. Muševič, Phys. Rev. Lett. 99, 247801 (2007).

[8] M. Ravnik and S. Žumer, Soft Matter 5, 4520 (2009).

[9] S. Žumer, "Modeling of constrained nematic order: from defects to colloidal structures," Plenary talk at $21^{\text {st }}$ Int. Liquid Crystal Conf., Keystone, Colorado (2006).

[10] T. Araki and H. Tanaka, Phys. Rev. Lett. 97, 127801 (2006).

[11] M. Ravnik and S. Žumer, Soft Matter 5, 269 (2009).

[12] N. D. Mermin, Rev. Mod. Phys. 51, 591 (1979).

[13] U. Tkalec, M. Ravnik, S. Čopar, S. Žumer, and I. Muševič, "Knots and links in chiral nematic colloids reconfigurable by light," To be published.

[14] S. Kutter, Elasticity of polymers with internal topological constraints, Ph.D. thesis, University of Cambridge (2002).

[15] R. D. Kamien, Eur. Phys. J. B 1, 1 (1998).

[16] F. B. Fuller, Proc. Nat. Acad. Sci. U.S.A. 75, 3557 (1978).

[17] D. Han, S. Pal, Y. Liu, and H. Yan, Nature Nanotech.
5, $712(2010)$.

[18] W. T. M. Irvine and D. Bouwmeester, Nat. Phys. 4, 716 (2008).

[19] J. E. Avron, D. Osadchy, and R. Seiler, Phys. Today 56, 38 (2003).

[20] R. D. Kamien, Rev. Mod. Phys. 74 (2002).

[21] M. A. Berger and C. Prior, J. Phys. A: Math. Gen. 39 (2006).

[22] V. V. Prasolov and A. B. Sossinsky, Knots, Links, Braids and 3-Manifolds (American Mathematical Society, Providence, Rhode Island, 1997) p. 32.

[23] K. Jänich, Acta Appl. Math. 8, 65 (1987).

[24] J. Fukuda, Phys. Rev. E 81, 040701 (2010).

[25] D. Kang, J. E. Maclennan, N. A. Clark, A. A. Zakhidov, and R. H. Baughman, Phys. Rev. Lett. 86, 4052 (2001).

[26] M. Otto and T. A. Vilgis, Phys. Rev. Lett. 80, 881 (1998).

[27] M. R. Dennis and J. H. Hannay, Proc. R. Soc. A 461, 3245 (2005).

[28] F. B. Fuller, Proc. Nat. Acad. Sci. U.S.A. 86, 815 (1971).

[29] J. H. White, Am. J. Math. 91, 693 (1969). 